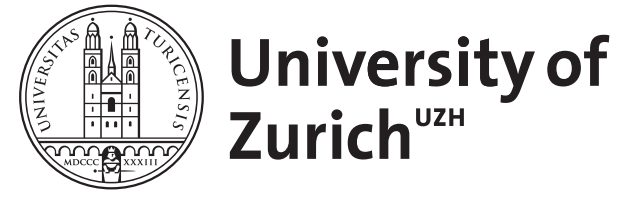

\title{
Hausärztlicher Notdienst - eine Frage der Kosten
}

Djalali, S ; Chmiel, C

\begin{abstract}
Dass die Notfallstationen von Spitälern chronisch überlastet sind, ist kein Geheimnis. Eine Grosszahl der dort vorstelligen Patienten könnte auch ambulant von hausärztlichen Notdiensten behandelt werden. Dies ist nicht nur praktisch, sondern auch kostengünstig, wie eine Studie in der Stadt Zürich zeigen konnte.
\end{abstract}

Posted at the Zurich Open Repository and Archive, University of Zurich ZORA URL: https://doi.org/10.5167/uzh-79788

Journal Article

Published Version

Originally published at:

Djalali, S; Chmiel, C (2013). Hausärztlicher Notdienst - eine Frage der Kosten. PrimaryCare, 13(13):235236. 


\section{Hausärztlicher Notdienst - eine Frage der Kosten}

Dass die Notfallstationen von Spitälern chronisch überlastet sind, ist kein Geheimnis. Eine Grosszahl der dort vorstelligen Patienten könnte auch ambulant von hausärztlichen Notdiensten behandelt werden. Dies ist nicht nur praktisch, sondern auch kostengünstig, wie eine Studie in der Stadt Zürich zeigen konnte.

Wenn Patienten sich ausserhalb der regulären Sprechzeiten an Notfallstationen von Spitälern wenden, ist das kostenintensiv. Längst liegen Studienergebnisse vor, die zeigen, dass ein Grossteil der medizinischen Probleme, mit denen die Patienten vorstellig werden, auch ambulant von einem Hausarzt behandelt werden könnte [1, 2]. Welche Kosten damit einhergehen, war die Frage in der hier vorgestellten Studie, die vom Institut für Hausarztmedizin Zürich in Zusammenarbeit mit dem Institut für Gesundheitsökonomie der Zürcher Hochschule für angewandte Wissenschaften in Winterthur durchgeführt wurde.

\section{Notfalldienst unter der Lupe}

685 Arzt-Patienten-Kontakte, die im Rahmen von hausärztlichen Notdiensten in der Stadt Zürich stattfanden, wurden im Rahmen dieser Studie ausgewertet. Dokumentiert wurden neben dem Behandlungsanlass die Art des Kontakts (Hausbesuch, Praxiskonsultation oder Telefonberatung) sowie die eingesetzten Ressourcen für die Behandlung (Zeit, diagnostische und therapeutische Massnahmen). Untersucht wurde sodann, welche Kosten durch die hausärztliche Behandlung für die Krankenkassen entstanden (Basis: TARMED und Eidgenössische Analyseliste).

\section{Kosten unter der Lupe}

Hierfür war wichtig, zwischen Basis- und Individualkosten zu differenzieren: Unter Basiskosten werden die fälligen Zeitpauschalen und Notfallzuschläge verstanden. Individualkosten sind dagegen die Kosten für die klinische Untersuchung, sowie vom Hausarzt angeordnete resp. durchgeführte diagnostische und therapeutische Massnahmen. Im Idealfall sind die durchschnittlichen Individualkosten niedrig, weil kostenintensive weiterführende Diagnostik und Therapie durch die hausärztliche Versorgung begrenzt werden können.

\begin{abstract}
Untersuchung und Behandlung schlagen kaum zu Buche Um die internationale Vergleichbarkeit zu gewährleisten, wurden die Kosten in Euro berechnet (1 CHF = 0,67 EUR).

Die durchschnittlichen Gesamtkosten für eine Notfallbehandlung betrugen 144 EUR. Wie sich in dieser Studie zeigte, trugen die durchschnittlichen Individualkosten tatsächlich am wenigsten zu den Gesamtkosten bei - unabhängig davon, ob es sich um einen Hausbesuch oder eine Praxiskonsultation handelte, machten die Untersuchungs- und Therapiekosten nur rund 30\% der Gesamtkosten aus. Demnach verursachten die diensthabenden Hausärzte bei einem Hausbesuch durchschnittlich 30 EUR an Individualkosten resp. 27 EUR im Rahmen einer Praxiskonsultation. Das Kostenmuster war unabhängig von der zugrundeliegenden Diagnose.
\end{abstract}

\section{Hausbesuch versus Telefonberatung}

Ausschlaggebend für die Höhe der Gesamtkosten war die Art der Konsultation: Bei Hausbesuchen (61\% aller untersuchten Konsul-

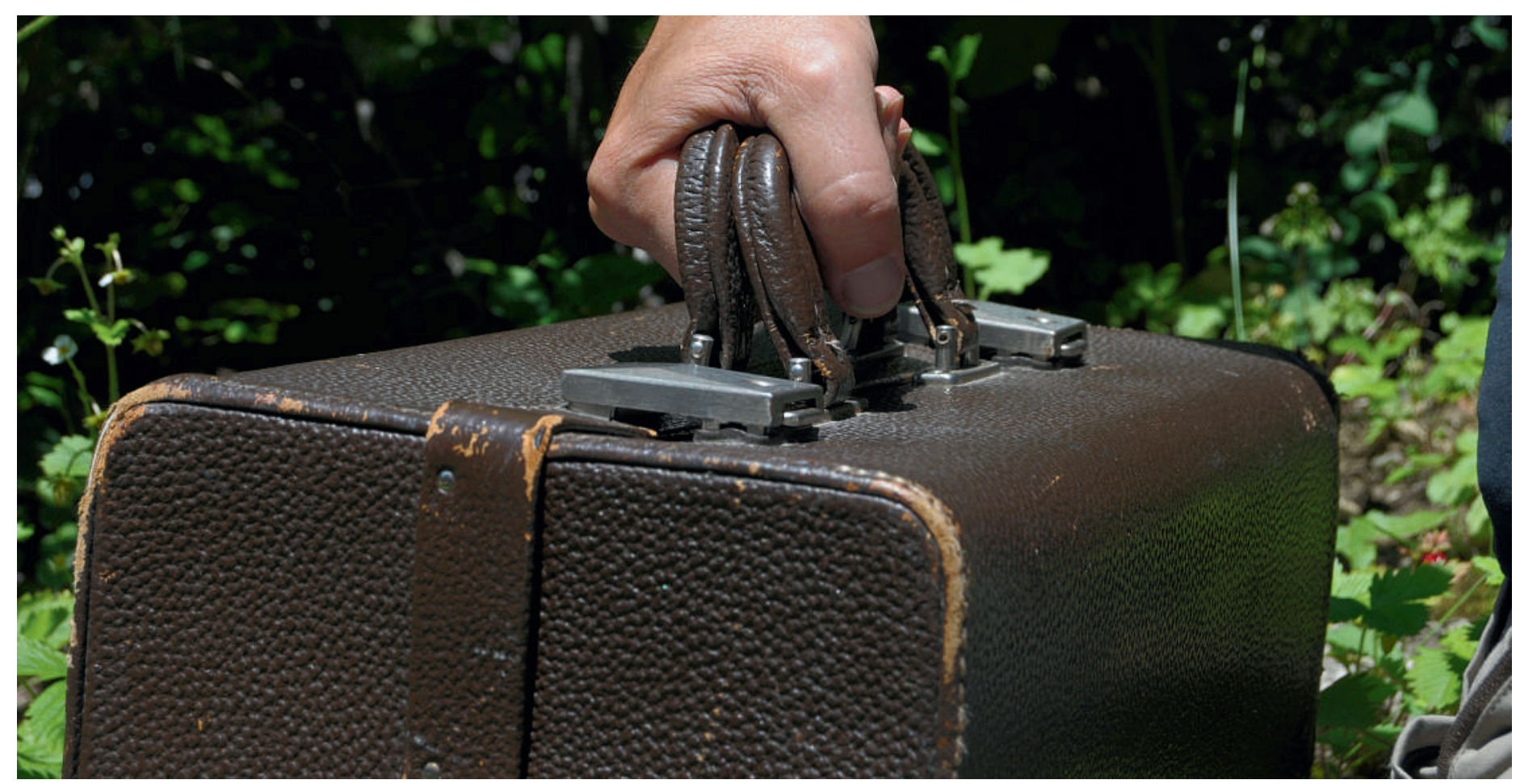

Allzeit bereit für eine effiziente Versorgung ausserhalb der Sprechzeiten - was ein einzelner Hausarzt heute selten leisten kann, schaffen hausärztliche Notfalldienste und entlasten dadurch die Spitäler. 
tationen; Gesamtkosten 176 EUR) betrugen die Basiskosten rund 146 EUR und damit 83\% der Gesamtkosten. Bei Praxiskonsultationen (25\% aller Kontakte; Gesamtkosten 90 EUR) betrugen die Basiskosten 63 EUR (70\% der Gesamtkosten). Reine Telefonberatungen (14\% aller Kontakte) waren mit durchschnittlichen Gesamtkosten von 48 EUR am günstigsten.

\section{Was lernen wir daraus?}

Die überwiegende Mehrheit (82\%) der Behandlungen in dieser Studie konnte ambulant abgeschlossen werden.

Insgesamt zeigt diese Studie, dass es den Hausärzten gelungen ist, die Notfallbehandlung ausserhalb der regulären Sprechstunden zu gewährleisten ohne zusätzlich zu den Basiskosten einen bedeutenden Aufwand an Individualkosten zu generieren - ein Ergebnis, das nicht nur der nationalen Gesundheitspolitik zu denken geben sollte, sondern auch durchaus international von Bedeutung ist überall dort, wo Vergütungssysteme von hausärztlichen Notdiensten zur Debatte stehen. Zukünftige Vergütungssysteme im Gesundheitswesen sollten hausärztliche Notdienste als kostengünstiges Versorgungsinstrument berücksichtigen und mehr finanzielle Anreize zum Aufbau und Betrieb solcher Notdienstangebote setzen.

\section{Literatur}

1 Eichler K, Imhof D, Chmiel C, Zoller M, Senn O, Rosemann T, Huber CA: The provision of out-of-hours care and associated costs in an urban area of Switzerland: a cost description study. BMC Fam Pract. 2010 Dec 20;11:99.
2 Chmiel C, Huber CA, Rosemann T, Zoller M, Eichler K, Sidler P, Senn O: Walk-ins seeking treatment at an emergency department or general practitioner out-of-hours service: a cross-sectional comparison. BMC Health Serv Res 2011;11:94.

Korrespondenz:

Dr. med. Sima Djalali

Institut für Hausarztmedizin, Universität Zürich

Pestalozzistrasse 24

8091 Zürich

sima.djalali[at]usz.ch

\section{PrimaryResearch - das Fenster zur Forschung}

In einer Artikelserie stellen wir Forschungsarbeiten aus dem Institut für Hausarztmedizin an der Universität Zürich (IHAMZ) vor. Die Originalarbeiten sind entweder «open access» zugänglich oder beim jeweiligen Autor auf Anfrage erhältlich. Die Ergebnisse geben einen spannenden Einblick in die täglichen Herausforderungen, aber auch die Leistung der Hausarztmedizin. An dieser Stelle ein herzlicher Dank an alle Kolleginnen und Kollegen, die sich an den Projekten beteiligen und die nachfolgenden Ergebnisse erst ermöglicht haben!

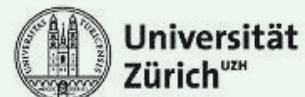

Institut für Hausarztmedizin 